\title{
Assessment of left ventricular systolic and diastolic abnormalities in patients with hypertrophic cardiomyopathy using real- time three-dimensional echocardiography and two-dimensional speckle tracking imaging
}

Xin Huang ${ }^{1 \dagger}$, Yan Yue ${ }^{2 \dagger}$, Yinmeng Wang ${ }^{3}$, Yujiao Deng ${ }^{4}$, Lu Liu' ${ }^{1}$ Yanqi Di ${ }^{1}$, Shasha Sun ${ }^{1}$, Deyou Chen ${ }^{5}$, Li Fan ${ }^{1}$ and Jian $\mathrm{CaO}^{1 *}$ (D)

\begin{abstract}
Background: Conventional echocardiography is not sensitive enough to assess left ventricular (LV) dysfunction in hypertrophic cardiomyopathy (HCM) patients. This research attempts to find a new ultrasonic technology to better assess LV diastolic function, systolic function, and myocardial longitudinal and circumferential systolic strain of segments with different thicknesses in HCM patients.

Methods: This study included 50 patients with HCM and 40 healthy subjects as controls. The peak early and late mitral annulus diastolic velocities at six loci $\left(E_{a}{ }^{\prime}\right.$ and $A_{a}{ }^{\prime}$, respectively) and the $E_{a}{ }^{\prime} / A_{a}{ }^{\prime}$ ratio were measured using real-time tri-plane echocardiography and quantitative tissue velocity imaging (RT-3PE-QTVI). The mean value of $E_{a}{ }^{\prime}$ at six loci $\left(E_{m}{ }^{\prime}\right)$ was obtained for the calculation of $E / E_{m}{ }^{\prime}$ ratio. The $L V$ end-diastolic volume (LVEDV), LV end-systolic volume (LVESV), LV stroke volume (LVSV), and LV ejection fraction (LVEF) were measured using real-time three-dimensional echocardiography (RT-3DE). LV myocardial longitudinal peak systolic strain (LPSS) and circumferential peak systolic strain (CPSS) in the apical-middle-basal segments (LPSS api, ${\text { LPSS-mid, } \text { LPSS }_{\text {-basi }} \text { CPSS }}_{\text {-api, }}$ CPSS $_{\text {-mid, }}$ and CPSS $_{\text {-bas, }}$ respectively) were obtained using a software for twodimensional speckle tracking imaging (2D-STI). According to the different segmental thicknesses (STs) in each HCM patient, the values (LPSS and CPSS) of all the myocardial segments were categorized into three groups and the respective averages were computed.

Results: The $E_{a}{ }^{\prime}, A_{a}{ }^{\prime}$, and, $E_{a}{ }^{\prime} / A_{a}{ }^{\prime}$ ratio in HCM patients were lower than those in the controls (all $p<0.001$ ), while the $E / E_{m}{ }^{\prime}$ ratio in HCM patients was higher than that in the controls $(p<0.001)$. The LVEDV, LVSV, and LVEF were significantly lower in HCM patients than in controls (all $p<0.001$ ). In HCM patients, the LPSS-api, LPSS $_{\text {-mid, }}$ LPSS $_{\text {-bas, }}$ CPSS -api, CPSS -mid, and CPSS -bas and the LPSS and CPSS of LV segments with different thicknesses were all significantly reduced (all $p<0.001$ ).

(Continued on next page)
\end{abstract}

* Correspondence: calvin301@163.com

${ }^{+}$Xin Huang and Yan Yue contributed equally to this work.

'Department of cardiology, Nanlou Division, Chinese PLA General Hospital,

National Clinical Research Center for Geriatric Diseases, Beijing 100853, China

Full list of author information is available at the end of the article

(c) The Author(s). 2018 Open Access This article is distributed under the terms of the Creative Commons Attribution 4.0 International License (http://creativecommons.org/licenses/by/4.0/), which permits unrestricted use, distribution, and reproduction in any medium, provided you give appropriate credit to the original author(s) and the source, provide a link to the Creative Commons license, and indicate if changes were made. The Creative Commons Public Domain Dedication waiver (http://creativecommons.org/publicdomain/zero/1.0/) applies to the data made available in this article, unless otherwise stated. 
(Continued from previous page)

Conclusions: In HCM patients, myocardial dysfunction was widespread not only in the obviously hypertrophic segments but also in the non-hypertrophic segments; the LV systolic and diastolic functions were damaged, even with a normal LVEF. LV diastolic dysfunction, systolic dysfunction, and myocardial deformation impairment in HCM patients can be sensitively revealed by RT-3PE-QTVI, RT-3DE, and 2D-STI.

Keywords: Hypertrophic cardiomyopathy, Left ventricular, Real-time tri-plane echocardiography and quantitative tissue velocity imaging, Real-time three-dimensional, Two-dimensional speckle tracking imaging, Systolic functions, Diastolic functions

\section{Background}

Hypertrophic cardiomyopathy (HCM) is an autosomal dominant genetic disease caused by a genetic mutation in the myocardial sarcomere gene, and is characterized by myocardial hypertrophy, interstitial fibrosis, myocyte disarray, and asymmetrical left ventricular (LV) hypertrophy [1, 2]. Conventional echocardiography has been used to diagnose diastolic dysfunction, which is characterized by impaired left ventricular relaxation with increased stiffness of the left ventricle and diminished filling rates in HCM patients. However, systolic dysfunction cannot be easily determined using conventional methods. Therefore, we often describe systolic function as normal or hyperdynamic in HCM patients, depending on normal or supernormal LV ejection fraction (LVEF). HCM patients often experience symptoms such as breathlessness, fatigue, and reduced exercise capacity [3]. Previously, it was believed that diastolic dysfunction is the predominant cause of these symptoms. However, molecular studies have confirmed that in HCM, cardiac contractile dysfunction occurs before myocardial hypertrophy $[4,5]$. Further, several studies have shown that the contractile force of the cardiac myocytes is impaired, despite the apparently normal or enhanced LVEF observed on conventional echocardiography $[6,7]$. Therefore, it is important to evaluate the cardiac function of HCM patients using some novel techniques. We designed this study with the aim of quantifying systolic dysfunction and myocardial deformation impairment in HCM patients using real-time three-dimensional echocardiography (RT-3DE) and two-dimensional speckle tracking imaging (2D-STI). Furthermore, LV diastolic function can be evaluated easily and quickly by measuring the mitral valve flow spectrum, but some patients in the pseudonormalization spectrum cannot be clearly assessed by traditional ultrasound technology. Therefore, in this study, measurement of diastolic velocity at six loci in mitral annulus using real-time tri-plane echocardiography and quantitative tissue velocity imaging (3PE-QTVI) was designed for the evaluation of LV diastolic function.

\section{Methods \\ Study population}

The study population included 50 patients with non-obstructive HCM, and they were consecutively selected. These patients had undergone clinical and echocardiographic evaluations at our hospital between October 2016 and November 2017. The inclusion criteria [8] were as follows: (1) A 2D ultrasound showing asymmetric hypertrophy in the LV walls; (2) septum/ posterior wall ratio $\geq 1.5$; and (3) inter-ventricular septum thickness $\geq 1.5 \mathrm{~mm}$. The exclusion criteria were as follows: (1) ventricular hypertrophy caused by simple apical HCM; (2) resting pressure gradient of the LV outflow tract $\geq 30 \mathrm{mmHg}$; (3) end-stage HCM (LVEF < $50 \%)$; (4) history of invasive septal reduction therapy (septal myomectomy or alcohol septal ablation); (5) organic (degenerative or rheumatic) valvular disease; (6) myocardial ischemia noted during noninvasive investigation, suggesting coronary artery disease; and (7) prior history of myocardial infarction or myocarditis. According to the segmental thickness (ST) in the diastole, all the segments of HCM patients were divided into three groups: HCM-A group ( $\mathrm{ST}<12 \mathrm{~mm} ; 227$ segments), HCM-B group (ST, 12-15 mm; 281 segments), and HCM-C group (ST > $15 \mathrm{~mm}$; 392 segments). For the healthy control group, 40 age- and gender-matched participants were selected from the same time frame based on the following conditions: (1) normal results on physical examination, echocardiography, electrocardiography (ECG), and biochemistry tests and (2) no coronary artery disease, hypertension, valvular disease, arrhythmia, and systemic disease.

\section{Prepared apparatus}

Conventional echocardiography and 2D-STI were performed in all participants, using the Vivid 7-dimension ultrasound machine and an M3S probe. The 3PE-QTVI and RT-3DE examinations were performed using a $3 \mathrm{~V}$ probe.

\section{Standard ultrasound examination}

The LV end-diastolic diameter (LVDd), LV end-systolic diameter (LVDs), LV posterior wall thickness (LVPWT), 
inter-ventricular septal thickness (IVST), and left atrial end-systolic dimension (LADs) were measured along the LV long-axis view. The IVST/LVPWT ratio was calculated. The pulsed wave sampling volume was placed under the tips of the mitral valve leaflets from the apical four-chamber view. The peak early and late mitral inflow diastolic velocities ( $\mathrm{E}$ and $\mathrm{A}$, respectively) were measured using pulse Doppler, and the E/A ratio was calculated.

\section{PE-QTVI examination}

During 3PE-QTVI, LV apical (long-axis and four- and two-chamber) views were obtained synchronously in cardiac cycles. The sampling volume was placed at six loci in the mitral annulus, namely, anterior septum, anterior wall, anterolateral wall, inferolateral wall, inferior wall, and inferior septum. The peak early and late mitral annulus diastolic velocity $\left(E_{a}{ }^{\prime}\right.$ and $A_{a}{ }^{\prime}$, respectively) and $E_{a}{ }^{\prime} / A_{a}^{\prime}$ ratio were measured by 3PE-QTVI. The mean value of $E_{a}{ }^{\prime}$ at the six loci $\left(E_{m}\right.$ ') was obtained for the calculation of $E / E_{m}$ '.

\section{RT-3DE examination}

RT-3DE full volume images were acquired during an end-expiratory breath-hold using a wide-angle acquisition mode in which four wedge-shaped subvolumes were obtained from four consecutive cardiac cycles with the acquisition triggered to the R wave of the ECG; subsequently, these datasets were stored and transferred to a computer for offline analysis. LV end-diastolic volume (LVEDV), LV end-systolic volume (LVESV), LV stroke volume (LVSV), and LVEF were calculated using a four-dimensional LV analysis software.

\section{D-STI examination}

High frame rate images of the LV long-axis, four- and two-chamber, and short-axis views of the basal, middle, and apical segments were obtained, respectively. Three consecutive cardiac cycles were acquired in each view and saved in a cine-loop format for offline analysis supported by a digitized software package. The software package automatically tracked the changes in the width of the region of interest, which was adjusted, as required, to fit the wall thickness. The automatic algorithm obtained an 18-segment model and provided the longitudinal peak systolic strain (LPSS) and circumferential peak systolic strain (CPSS) for each segment. Subsequently, the average LPSS and CPSS values of basal, middle, and apical segments

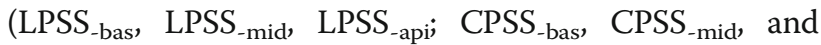
$\mathrm{CPSS}_{\text {-api }}$, respectively) were calculated. The values (LPSS and CPSS) of all the myocardial segments for each HCM patient were categorized into three groups (HCM-A,
HCM-B, and HCM-C) according to the ST, and subsequently, the average was computed.

The aforementioned ultrasound parameters were measured according to the American Society of Echocardiography guidelines and requirements [9].

\section{Statistical analysis}

Data analysis was performed using the Statistical Package for the Social Sciences software (SPSS, Version 19.0). The results are presented as mean \pm standard deviation. The normality test was used to compare continuous variables, and the one-way analysis of variance was used to compare the echocardiographic values in HCM patients and the control group. Continuous variables obtained from different groups were compared using the Fisher's least significant difference test. All the $p$ values were two-sided, and $p<0.05$ was considered statistically significant.

\section{Results}

\section{General information}

The clinical characteristics of HCM patients and the control group are shown in Table 1. Both the groups were well matched with respect to age and gender. No statistical differences were observed in the body surface area, heart rate, and blood pressure between the two groups (all $p>0.05$ ).

\section{Conventional echocardiographic parameters}

The IVST, LVPWT, septum/posterior wall ratio, and LADs were significantly higher in HCM patients than in the control group (all $p<0.001$ ), whereas LVDd and LVDs were significantly lower in HCM patients than in the control group (all $p<0.001$ ). Transmitral Doppler measurements showed no significant difference between the peak E, peak $\mathrm{A}$, and $\mathrm{E} / \mathrm{A}$ in $\mathrm{HCM}$ patients and those in the control group (all $p>0.05$ ) (Table 1).

\section{PE-QTVI parameters}

In this study, $\mathrm{E}_{\mathrm{a}}{ }^{\prime}, \mathrm{A}_{\mathrm{a}}{ }^{\prime}$, and $\mathrm{E}_{\mathrm{a}}{ }^{\prime} / \mathrm{A}_{\mathrm{a}}{ }^{\prime}$ ratio at the six loci in the mitral annulus in HCM patients were lower than those in the control group, whereas the $E / E_{m}{ }^{\prime}$ ratio in HCM patients was higher than that in the control group; all the differences were statistically significant (all $p<0.001$ ) (Table 2; Fig. 1).

\section{RT-3DE parameters}

RT-3DE showed that LVEDV, LVSV, and LVEF were significantly lower in HCM patients than in the control group (all $p<0.001$ ), although LVEF was within the normal range (LVEF $>50 \%$ ) (Table 3; Fig. 2). 
Table 1 Clinical characteristics and conventional echocardiographic parameters in HCM patients versus control group

\begin{tabular}{|c|c|c|c|}
\hline & HCM patients $(N=50)$ & Control group $(N=40)$ & $p$ value \\
\hline Age(years) & $46.67 \pm 11.09$ & $43.93 \pm 8.17$ & 0.195 \\
\hline \multicolumn{4}{|l|}{ Gender } \\
\hline Male & $30(60 \%)$ & $24(60 \%)$ & \\
\hline Female & $20(40 \%)$ & $16(40 \%)$ & \\
\hline HR(beats/min) & $70.51 \pm 9.62$ & $68.43 \pm 11.95$ & 0.362 \\
\hline $\mathrm{BSA}\left(\mathrm{m}^{2}\right)$ & $1.72 \pm 0.15$ & $1.70 \pm 0.21$ & 0.612 \\
\hline $\mathrm{SBP}(\mathrm{mmHg})$ & $124.63 \pm 13.05$ & $120.59 \pm 9.67$ & 0.106 \\
\hline $\mathrm{DBP}(\mathrm{mmHg})$ & $78.32 \pm 11.57$ & $74.81 \pm 9.07$ & 0.119 \\
\hline IVST (mm) & $25.33 \pm 7.11$ & $9.32 \pm 1.35$ & $<0.001$ \\
\hline LVPWT (mm) & $14.25 \pm 3.15$ & $8.84 \pm 1.11$ & $<0.001$ \\
\hline septum/posterior wall ratio & $1.86 \pm 0.69$ & $1.05 \pm 0.10$ & $<0.001$ \\
\hline LVDd (mm) & $40.83 \pm 4.87$ & $44.16 \pm 2.44$ & $<0.001$ \\
\hline LVDs (mm) & $23.32 \pm 4.09$ & $27.81 \pm 1.64$ & $<0.001$ \\
\hline LADs & $40.91 \pm 0.83$ & $31.85 \pm 1.95$ & $<0.001$ \\
\hline $\mathrm{E}(\mathrm{cm} / \mathrm{sec})$ & $79.04 \pm 3.26$ & $80.36 \pm 3.07$ & 0.053 \\
\hline $\mathrm{A}(\mathrm{cm} / \mathrm{sec})$ & $71.05 \pm 2.89$ & $69.89 \pm 2.65$ & 0.052 \\
\hline E / A ratio & $1.12 \pm 0.39$ & $1.15 \pm 0.29$ & 0.675 \\
\hline
\end{tabular}

$H R$ heart rate, $B S A$ body surface area, SBP systolic blood pressure, DBP diastolic blood pressure, IVST inter-ventricular septal thickness, LVDd left ventricular enddiastolic diameter, LVDs left ventricular end-systolic diameter, LVPWT left ventricular posterior wall thickness, LADs left atrium end-systolic dimension. E: mitral inflow peak velocity in early diastole; A: mitral inflow peak velocity in late diastole. Values are expressed as the mean \pm SD

\section{D-STI parameters}

A gradual increase in the amplitudes of LPSS and CPSS was observed from the basal segment to the apex in the control group. However, in HCM patients, this regular pattern was not evident, and LPSS $_{\text {-bas, }}$, LPSS $_{\text {-mid }}$, LPSS $_{\text {-api }}$, CPSS $_{\text {-bas }}$, CPSS $_{\text {-mid }}$, and CPSS decreased significantly (all $p<0.001$ ) (Table 4; Figs. 3 and 4). LPSS and CPSS of the LV myocardial segments in HCM-A, HCM-B, and HCM-C groups were all reduced significantly compared with those in the control group (all $p<0.001$ ), and there was a statistically significant difference between LPSS and CPSS of the LV myocardial segments among HCM-A, HCM-B, and HCM-C groups (all $p<0.001$ ); a decreased amount of strain correlated with the myocardial thickness grade (Table 5). There was a statistically significant difference in the thickness of the myocardium among HCM-B, HCM-C groups and the control group (all $p<0.001$ ); there was no significant difference in the thickness of the myocardium between HCM-A and the control group.

\section{Discussion}

Owing to the complexity of a three-dimensional cardiac structure, helical ventricular myocardial band (HVMB) is considered to be a critical cornerstone in understanding cardiac function. The HVMB comprises a helical component, namely an apical vortex (oblique descending and ascending segments that cross each other at $60^{\circ}$ angles en-route to forming) and a basal loop (containing both left and right transverse sides) [10]. During ventricular contraction, all the components coordinate synergistically to achieve myocardial longitudinal shortening, circumferential narrowing or compression, radial thickening, and twisting in favor of ejection. Because of mutation in the genes encoding the sarcomeric protein of the cardiac contractile apparatus, a series of characteristic pathological changes occur in HCM patients. This study aimed to quantify the effects of the pathological changes on the LV systolic and diastolic functions in HCM patients.

\section{Evaluation of LV diastolic function in HCM patients by 3PE-QTVI}

The Moderate reduction of diastolic function, usually showing E/A ratio $>1$, is due a pseudonormalized phenomenon, which is difficult to distinguish from normal cardiac function. In this study, E and A were not significantly different between HCM patients and the control group, and the E/A ratio was $>1$, which means that the mitral valve orifice flow spectrum may not truly reflect the changes in the diastolic function in patients with HCM.

The present study reported that the measurement of early and late mitral annulus diastolic velocity can reflect changes in relaxation and compliance of the left 
Table 2 RT-3PE-QTVI parameters in HCM patients versus control group

\begin{tabular}{|c|c|c|c|}
\hline & HCM patients $(N=50)$ & Control group $(N=40)$ & $p$ value \\
\hline \multicolumn{4}{|l|}{$\mathrm{E}_{\mathrm{a}}^{\prime}(\mathrm{cm} / \mathrm{sec})$} \\
\hline AS & $3.35 \pm 1.15$ & $8.14 \pm 2.23$ & $<0.001$ \\
\hline AN & $3.58 \pm 1.25$ & $8.87 \pm 1.81$ & $<0.001$ \\
\hline $\mathrm{AL}$ & $4.24 \pm 1.50$ & $9.25 \pm 1.80$ & $<0.001$ \\
\hline $\mathbb{I L}$ & $3.88 \pm 1.44$ & $10.26 \pm 2.01$ & $<0.001$ \\
\hline IN & $3.51 \pm 1.46$ & $9.18 \pm 1.57$ & $<0.001$ \\
\hline IS & $3.44 \pm 1.42$ & $8.20 \pm 1.31$ & $<0.001$ \\
\hline \multicolumn{4}{|l|}{$A_{a}^{\prime}(\mathrm{cm} / \mathrm{sec})$} \\
\hline AS & $5.12 \pm 1.84$ & $6.48 \pm 0.87$ & $<0.001$ \\
\hline AN & $5.22 \pm 1.20$ & $7.08 \pm 0.56$ & $<0.001$ \\
\hline $\mathrm{AL}$ & $5.93 \pm 1.43$ & $7.21 \pm 0.65$ & $<0.001$ \\
\hline $\mathrm{IL}$ & $6.02 \pm 1.58$ & $7.59 \pm 1.13$ & $<0.001$ \\
\hline IN & $5.68 \pm 1.46$ & $7.80 \pm 1.32$ & $<0.001$ \\
\hline IS & $5.10 \pm 1.27$ & $6.63 \pm 0.68$ & $<0.001$ \\
\hline \multicolumn{4}{|l|}{$E_{a}^{\prime} / A_{a}^{\prime}$} \\
\hline AS & $0.71 \pm 0.26$ & $1.27 \pm 0.43$ & $<0.001$ \\
\hline AN & $0.69 \pm 0.20$ & $1.24 \pm 0.18$ & $<0.001$ \\
\hline $\mathrm{AL}$ & $0.74 \pm 0.28$ & $1.29 \pm 0.23$ & $<0.001$ \\
\hline IL & $0.63 \pm 0.22$ & $1.45 \pm 0.33$ & $<0.00$ \\
\hline IN & $0.63 \pm 0.25$ & $1.21 \pm 0.29$ & $<0.001$ \\
\hline IS & $0.69 \pm 0.18$ & $1.26 \pm 0.32$ & $<0.00$ \\
\hline $\mathrm{E} / \mathrm{E}_{\mathrm{m}}{ }^{\prime}$ ratio & $21.47 \pm 5.36$ & $10.01 \pm 3.94$ & $<0.00$ \\
\hline
\end{tabular}

$E_{a}^{\prime}$ early diastolic velocity of mitral annulus, $A_{a}^{\prime}$ late-diastolic velocity of mitral annulus, $E$ mitral inflow peak velocity in early diastole, $E_{m}$ ' mean value of $E_{a}^{\prime}$ at six loci in mitral annulus, $A S$ anterior septum, $A N$ anterior wall, $A L$ anterolateral wall, IL inferolateral wall, IN inferior wall, IS inferior septum. Values are expressed as the mean $\pm \mathrm{SD}$

ventricle, independent of the cardiac load [3]. In this study, 3PE-QTVI was used for detection. By obtaining three orthogonal views from a $60^{\circ}$ angle synchronously, this technology overcomes the limitations of conventional QTVI, such as display of only one view in each
Table 3 RT-3DE parameters in HCM patients versus control group

\begin{tabular}{llll}
\hline & HCM patients $(\mathrm{N}=50)$ & Control group $(\mathrm{N}=40)$ & $p$ value \\
\hline $\operatorname{LVEDV}(\mathrm{ml})$ & $70.12 \pm 13.91$ & $85.59 \pm 14.94$ & $<0.001$ \\
$\operatorname{LVESV}(\mathrm{ml})$ & $31.09 \pm 6.02$ & $29.46 \pm 5.47$ & 0.187 \\
$\operatorname{LVSV}(\mathrm{ml})$ & $39.94 \pm 10.67$ & $56.25 \pm 11.68$ & $<0.001$ \\
$\operatorname{LVEF}(\%)$ & $57.17 \pm 4.15$ & $65.13 \pm 2.95$ & $<0.001$ \\
\hline
\end{tabular}

LVEDV left ventricular end-diastolic volume, LVESV left ventricular end-systolic volume, LVSV left ventricular stroke volume, LVEF left ventricular ejection

fraction. Values are expressed as the mean $\pm S D$

cardiac cycle and susceptibility to heart rate and breathing. In this study, $E_{a}{ }^{\prime}, A_{a}{ }^{\prime}$, and $E_{a}{ }^{\prime} / A_{a}{ }^{\prime}$ ratio in HCM patients were lower than those in the control group. The results showed that relaxation and compliance of the left ventricle were impaired. In addition, the $E / E_{m}$ ' ratio reflects LV end-diastolic pressure (LVEDP). The $E / E_{m}{ }^{\prime}$ ratio in HCM patients significantly increased compared with that in the control group, indicating that LVEDP was significantly higher in $\mathrm{HCM}$ patients. When the myocardium of the left ventricle (main pumping chamber) is of abnormal thickness, the myocardial stiffness increases and compliance decreases, which results in increase in LV filling pressure. Long-term restriction of left atrial to ventricular blood filling may lead to left atrial enlargement. Left atrial dilation negatively affects patient condition and prognosis and may lead to atrial fibrillation, heart failure, sudden death, and other cardiovascular events $[11,12]$.

\section{Evaluation of LV pumping function in HCM patients by RT-3DE}

RT-3DE can overcomes the limitations of 2D echocardiography by demonstrating a $3 \mathrm{D}$ ventricular shape at different time phases and depicting the whole extent of the ventricular endocardium. In this study, LVEDV, LVSV, and LVEF measured by RT-3DE were observed to have significantly reduced in HCM patients compared with those in the control group, although LVEF
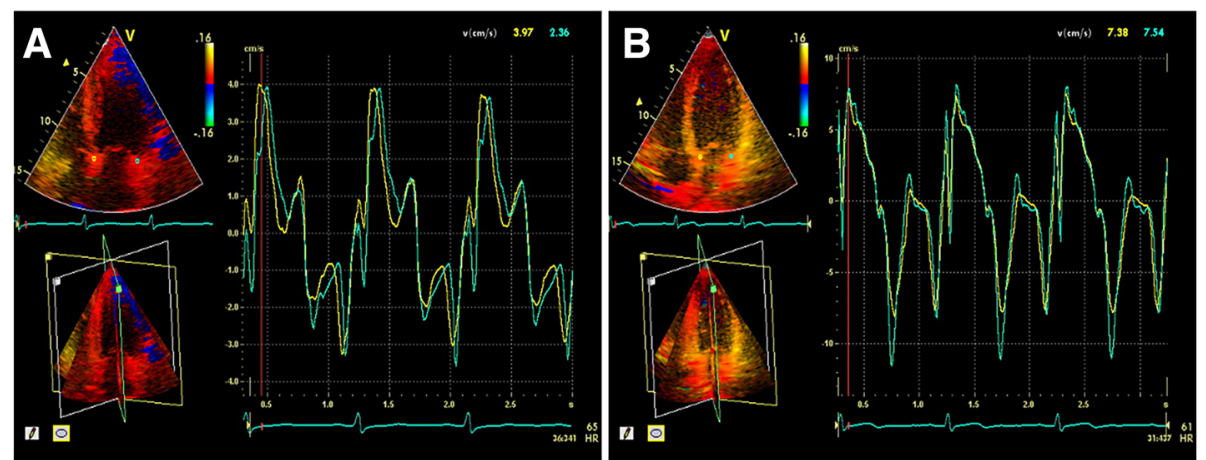

Fig 1. The peak early and late mitral annulus diastolic velocities (Ea' and $A a^{\prime}$, respectively) at apical four-chamber view were measured using real-time tri-plane echocardiography and quantitative tissue velocity imaging. Ea' and Aa' are significantly lower in HCM patients than in control group 

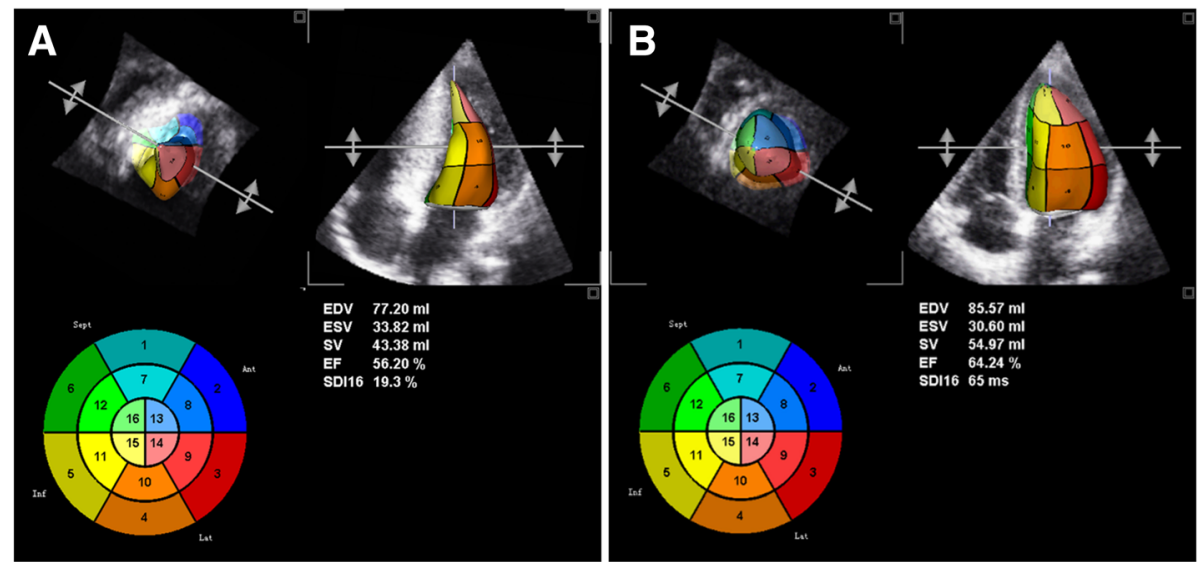

Fig 2. Left ventricular (LV) global shell maps by real-time three-dimensional echocardiography full volume image are shown. Left ventricle is seen as a "wedge-shape" in HCM patients. LV end-diastolic volume (LVEDV), end-systolic volume (LVESV), ejection fraction (LVEF) were significantly lower in HCM patients than in control group

was in the normal range (LVEF $>50 \%$ ). Ejection fraction, depending on the percentage of stroke volume in LVEDV, mainly reflects changes in the cardiac cavity dimensions and volume, and is obviously affected by cardiac preload and afterload. The hypertrophic myocardium and papillary muscles protrude into the chamber [13], and reduce LV dimensions and volume, which is seen as a wedge- or spade-shaped ventricular cavity in HCM patients. Therefore, LVEF cannot completely reflect the cardiac stroke volume, especially in HCM patients with obviously decreased LV dimension and volume. Merely applying the parameter to evaluate LV systolic function can cause errors or bias [14, 15]. This study showed that LVSV had significantly decreased, although LVEF was in the normal range (LVEF > 50\%). Hence, the LVSV may objectively and sensitively reveal the impairment of LV systolic function in HCM patients, despite a normal LVEF. We have previously demonstrated that symptoms such as syncope, palpitation, breathlessness, and exercise

Table 4 STI results of longitudinal and circumferential strain in $\mathrm{HCM}$ patients versus control group

\begin{tabular}{llll}
\hline & HCM patients $(\mathrm{N}=50)$ & Control group $(\mathrm{N}=40)$ & $p$ value \\
\hline LPSS $_{\text {-bas }}$ & $-13.91 \pm 5.17$ & $-21.67 \pm 3.50$ & $<0.001$ \\
LPSS $_{\text {-mid }}$ & $-12.59 \pm 5.83$ & $-22.02 \pm 3.06$ & $<0.001$ \\
LPSS $_{\text {-api }}$ & $-14.76 \pm 6.84$ & $-24.58 \pm 3.96$ & $<0.001$ \\
CPSS $_{\text {-bas }}$ & $-16.99 \pm 6.19$ & $-22.13 \pm 4.86$ & $<0.001$ \\
CPSS $_{\text {-mid }}$ & $-15.85 \pm 5.34$ & $-23.45 \pm 3.70$ & $<0.001$ \\
CPSS $_{\text {-api }}$ & $-16.24 \pm 4.58$ & $-28.32 \pm 4.15$ & $<0.001$ \\
\hline
\end{tabular}

LPSS longitudinal peak systolic strain, CPSS circumferential peak systolic strain. Values are expressed as the mean \pm SD limitation are closely related to diastolic dysfunction. In this study, we considered that those symptoms could also be indicative of reduced LVSV.

\section{Evaluation of LV myocardial deformation in HCM patients by 2D-STI}

In this study, LPSS and CPSS values in apical-middle-basal segments showed a regular pattern in the control group. The amplitudes of LPSS and CPSS increased gradually from the basal segment to the apical segment in the control group. However, this regularity was not evident in HCM patients. The LV LPSS and CPSS values of the basal, middle, and apical segments decreased significantly. This indicated that the LV myocardial systolic function was impaired in HCM patients. This impairment of the LV myocardial systolic function could be caused by the following factors: (1) HCM is characterized by abnormal hypertrophy in the cardiomyocytes, increased collagen, and interstitial fibrosis, and even macroscopically visible myocardial scarring [1, 16]; (2) hypertrophic myocardium and myofibrillar disarray are also considered pathological hallmarks of HCM, these are distributed unevenly and arranged irregularly in the form of swirls or clusters [1, 2, 16]; and (3) microvascular dysfunction occurs in HCM, which features myocardial/vascular ratio imbalance (decrease in myocardial capillary density), coronary artery wall thickening, and development of myocardial ischemia [17, 18].

Furthermore, in this study, LPSS and CPSS of the LV myocardial segments with different thicknesses were reduced significantly in HCM patients, even the normal thickness of the HCM-A group was significantly lower than that of the control group, but there 

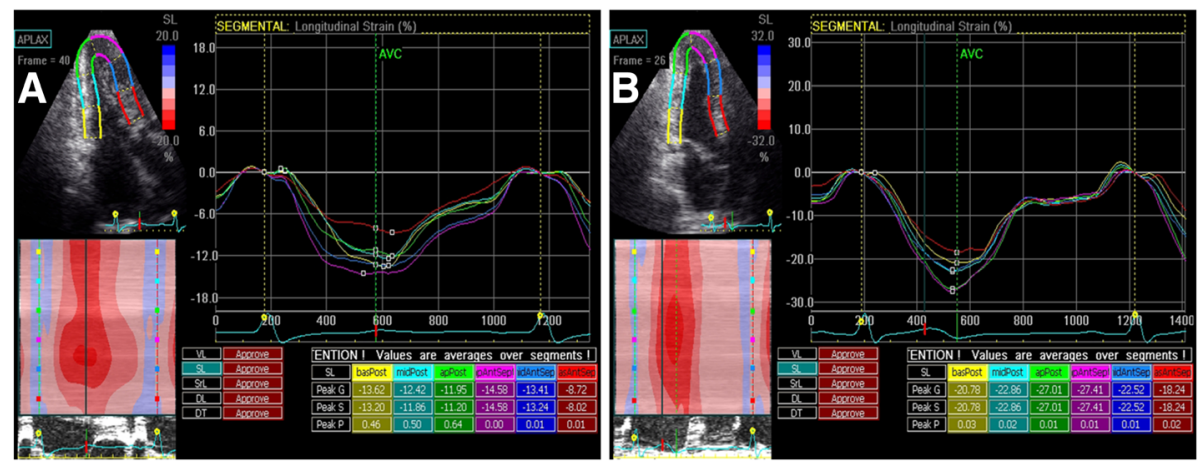

Fig 3. Two-dimensional speckle tracking images in left ventricular apical long-axis view are shown. Left ventricular longitudinal peak systolic strain in HCM patients is significantly lower than in control group

was no significant difference in the thickness of the myocardium between HCM-A and the normal control group. Further, the decreased amount of strain correlated with the myocardial thickness grade. Thus, the myocardial systolic dysfunction not only in the obvious hypertrophic segments but also in the non-hypertrophic segments suggested that myocardial systolic dysfunction in HCM patients may precede myocardial hypertrophy. This result is consistent with the results reported in previous studies [15]. Therefore, myocardial systolic dysfunction may occur first and the hypertrophy could be a compensatory response. The more obvious the magnitude of hypertrophy, the more serious is the impairment of myocardial strain. Just as demonstrated by Urbano-Moral et al. [19], the extent of hypertrophy is the primary factor altering myocardial mechanics. For HCM patients, the degree of myocardial hypertrophy is one of the parameters evaluated during regular follow-ups, and this study suggests that this evaluation may be important for assessing myocardial function, predicting the progress of the disease, and guiding therapy in clinical practice.

\section{Limitations}

Several limitations of this study must be considered. First, because the sample size was rather small, the results obtained from this study may not be statistically strong. Second, evaluation by RT-3DE and 2D-STI was significantly dependent on the quality of image. The assessments were feasible in patients with sufficient images quality. Some situations such as poor acoustic window (resulting in poor images) of cardiac patients with arrhythmia and examiner's subjective experience could have affected the accuracy and reliability of the data. In this study, the patients with poor images or arrhythmia were ruled out. Third, LVEF of HCM group is significantly lower than that of control group, even though it is within normal range. That can be a basic difference between two groups of this study, and consequently affect the other results comparing two groups. HCM group of this study may be patients with systolic dysfunction as well as diastolic dysfunction. Finally, a bias existed in different echocardiographic software packages from various ultrasound machine vendors, which may have affected the measurement repeatability and accuracy within acceptable limits.
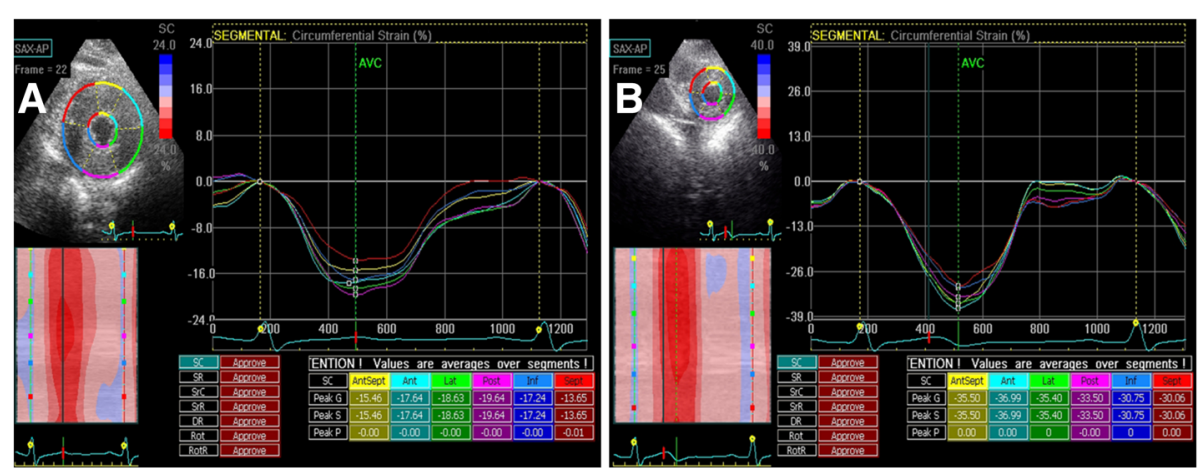

Fig 4. Two-dimensional speckle tracking images in left ventricular short-axis view at the apical level are shown. Left ventricular circumferential peak systolic strain is significantly lower in HCM patients than in control group 
Table 5 STI results of the peak systolic longitudinal and circumferential strain in different segmental thickness(ST) groups in HCM patients and control group

\begin{tabular}{lllll}
\hline & HCM-A (ST<12 mm, 227segments) & HCM-B (ST:12-15 mm, 281 segments) & HCM-C (ST>15 mm, 392 segments) & Control group (720 segments) \\
\hline ST & $9.45 \pm 1.25$ & $13.39 \pm 1.21^{* \#}$ & $17.53 \pm 1.84^{* \# \&}$ & $9.37 \pm 1.18$ \\
LPSS & $-17.79 \pm 3.75^{*}$ & $-13.51 \pm 4.02^{* \#}$ & $-10.03 \pm 3.17^{* \# \&}$ & $-22.89 \pm 3.41$ \\
CPSS & $-19.83 \pm 4.07^{*}$ & $-16.19 \pm 5.71^{* \#}$ & $-13.32 \pm 4.39^{* \# \&}$ & $-24.61 \pm 3.52$ \\
\hline
\end{tabular}

Values are expressed as the mean \pm SD

" $p<0.001$ vs. Control group' segment

$\# p<0.001$ vs. HCM-A group (ST $<12 \mathrm{~mm}$ )

$\&_{p}<0.001$ vs. HCM-B group (ST:12 mm $\left.-15 \mathrm{~mm}\right)$

\section{Conclusions}

This study shows that widespread myocardial dysfunction occurred not only in the obvious hypertrophic segments but also in the non-hypertrophic segments. The more obvious the magnitude of hypertrophy, the more serious is the impairment of myocardial strain. The LV systolic and diastolic functions were damaged; however, the LVEF in HCM patients was in the normal range. The LV diastolic dysfunction, systolic dysfunction, and myocardial deformation impairment in HCM patients can be early, objectively, and sensitively revealed by RT-3PE-QTVI, RT-3DE, and 2D-STI.

\section{Abbreviations}

2D-STI: Two-dimensional speckle tracking imaging; 3PE-QTVI: Real-time triplane echocardiography and quantitative tissue velocity imaging; $\mathrm{A}$ : The peak late mitral annulus diastolic velocities; $A_{a}$ : The peak late mitral annulus diastolic velocities at six loci; CPSS: Circumferential peak systolic strain; CPSS api: Circumferential peak systolic strain in the apical segments; CPSS bas: Circumferential peak systolic strain in the basal segments; CPSS mid: Circumferential peak systolic strain in the middle segments; E: The peak early mitral annulus diastolic velocities; $E_{a}^{\prime}$ : The peak early mitral annulus diastolic velocities at six loci; $E_{m}^{\prime}$ : The mean value of $E_{a}{ }^{\prime}$ at six loci; HCM: Hypertrophic cardiomyopathy; HCM-A: Segmental thickness $<12$ mm; HCM-B: Segmental thickness: 12-15 mm; HCM-C: Segmental thickness > 15 mm; HVMB: Helical ventricular myocardial band; IVST: Inter-ventricular septal thickness; LADs: Left atrial end-systolic dimension; LPSS: Longitudinal peak systolic strain; LPSS-api: Longitudinal peak systolic strain in the apical segments; LPSS-bas: Longitudinal peak systolic strain in the basal segments;

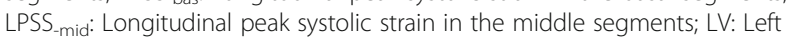
ventricular; LVDd: Left ventricular end-diastolic diameter; LVDs: Left ventricular end-systolic diameter; LVEDP: Left ventricular end-diastolic pressure; LVEDV: Left ventricular end-diastolic volume; LVEF: Left ventricular ejection fraction; LVESV: Left ventricular end-systolic volume; LVPWT: Left ventricular posterior wall thickness; LVSV: Left ventricular stroke volume; RT3DE: real-time three-dimensional echocardiography; ST: Segmental thickness

\section{Availability of data and materials}

The datasets used and/or analyzed during the current study are available from the corresponding author on reasonable request.

\section{Authors' contributions}

$\mathrm{XH}$ and $\mathrm{YY}$ as the co-first author, JC as the corresponding author: they designed this study and analyzed and interpreted the patient data, drafted the manuscript, control and guarantee that all aspects of the work were investigated and resolved. YW, YD, LL, YD, SS, DC, and LF were responsible for acquisition of data, analysis and interpretation of data, revising the manuscript, and they control and guarantee that all aspects of the work were investigated and resolved. All authors read and approved the final manuscript.

\section{Ethics approval and consent to participate}

This study has been approved by the Chinese PLA General Hospital Medica Ethics Committee and have therefore been performed in accordance with the ethical standards laid down in the 1964 Declaration of Helsinki and its later amendments. All persons gave their informed consent prior to their inclusion in the study.

\section{Consent for publication}

All the authors contributed substantially to the project and agreed for sending the manuscript to Cardiovascular Ultrasound. There is no competing interest and the data are available.

\section{Competing interests}

The authors declare that they have no conflict of interests.

\section{Publisher's Note}

Springer Nature remains neutral with regard to jurisdictional claims in published maps and institutional affiliations.

\section{Author details}

'Department of cardiology, Nanlou Division, Chinese PLA General Hospital, National Clinical Research Center for Geriatric Diseases, Beijing 100853, China. ${ }^{2}$ Department of medical administration, Chinese PLA General Hospital, Beijing 100853, China. ${ }^{3}$ Department of Respiration, Clifford Hospital, Guangzhou 511495, China. ${ }^{4}$ Department of Ultrasound, Chinese PLA General Hospital, Beijing 100853, China. ${ }^{5}$ Department of Outpatient, Chinese PLA General Hospital, Beijing 100853, China.

Received: 22 March 2018 Accepted: 5 September 2018

Published online: 02 October 2018

\section{References}

1. Marian AJ, Braunwald E. Hypertrophic cardiomyopathy: genetics, pathogenesis, clinical manifestations, diagnosis, and therapy. Circ Res. 2017; 121(7):749-70

2. Kocovski L, Fernandes J. Sudden cardiac death: a modern pathology approach to hypertrophic cardiomyopathy. Arch Pathol Lab Med. 2015; 139(3):413-6.

3. Abozguia K, Nallur-Shivu G, Phan TT, Ahmed I, Kalra R, Weaver RA, et al. Left ventricular strain and untwist in hypertrophic cardiomyopathy: relation to exercise capacity. Am Heart J. 2010;159(5):825-32.

4. Pislaru C, Anagnostopoulos PC, Seward JB, Greenleaf JF, Belohlavek M. Higher myocardial strain rates during isovolumic relaxation phase than during ejection characterize acutely ischemic myocardium. J Am Coll Cardiol. 2002;40(8):1487-94.

5. Rust EM, Albayya FP, Metzger JM. Identification of a contractile deficit in adult cardiac myocytes expressing hypertrophic cardiomyopathy-associated mutant troponin T proteins. J Clin Invest. 1999;103(10):1459-67.

6. Ozawa K, Funabashi N, Takaoka H, Kamata T, Kanaeda A, Saito M, et al. Characteristic myocardial strain identified in hypertrophic cardiomyopathy subjects with preserved left ventricular ejection fraction using a novel multi-layer transthoracic echocardiography technique. Int J Cardiol. 2015;184:237-43.

7. Marian AJ. Pathogenesis of diverse clinical and pathological phenotypes in hypertrophic cardiomyopathy. Lancet. 2000;355(9197):58-60.

8. Gersh BJ, Maron BJ, Bonow RO, Dearani JA, Fifer MA, Link MS, et al. 2011 ACCF/AHA Guideline for the Diagnosis and Treatment of Hypertrophic Cardiomyopathy: a report of the American College of Cardiology Foundation/American Heart Association Task Force on Practice Guidelines. 
Developed in collaboration with the American Association for Thoracic Surgery, American Society of Echocardiography, American Society of Nuclear Cardiology, Heart Failure Society of America, Heart Rhythm Society, Society for Cardiovascular Angiography and Interventions, and Society of Thoracic Surgeons. J Am Coll Cardiol. 2011;58(25):e212-60.

9. Lang RM, Bierig M, Devereux RB, Flachskampf FA, Foster E, Pellikka PA, et al. Recommendations for chamber quantification: a report from the American Society of Echocardiography's guidelines and standards committee and the chamber quantification writing group, developed in conjunction with the European Association of Echocardiography, a branch of the European Society of Cardiology. J Am Soc Echocardiogr. 2005;18(12):1440-63.

10. Buckberg $\mathrm{G}$. The helical ventricular myocardial band during standard echocardiography: a structure-function relationship. Echocardiography. 2015;32(2):199-204.

11. Parato VM, Antoncecchi V, Sozzi F, Marazia S, Zito A, Maiello M, et al. Echocardiographic diagnosis of the different phenotypes of hypertrophic cardiomyopathy. Cardiovasc Ultrasound. 2016:14(1):30.

12. Graca B, Ferreira MJ, Donato P, Castelo-Branco M, Caseiro-Alves F. Cardiovascular magnetic resonance imaging assessment of diastolic dysfunction in a population without heart disease: a gender-based study. Eur Radiol. 2014;24(1):52-9.

13. Zhang HJ, Wang H, Sun T, Lu MJ, Xu N, Wu WC, et al. Assessment of left ventricular twist mechanics by speckle tracking echocardiography reveals association between LV twist and myocardial fibrosis in patients with hypertrophic cardiomyopathy. Int J Cardiovasc Imaging. 2014;30(8):1539-48.

14. Yoshikawa H, Suzuki M, Hashimoto G, Kusunose Y, Otsuka T, Nakamura M, et al. Midwall ejection fraction for assessing systolic performance of the hypertrophic left ventricle. Cardiovasc Ultrasound. 2012;10:45.

15. Liu L, Tuo S, Zhang J, Zuo L, Liu F, Hao L, et al. Reduction of left ventricular longitudinal global and segmental systolic functions in patients with hypertrophic cardiomyopathy: study of two-dimensional tissue motion annular displacement. Exp Ther Med. 2014;7(6):1457-64.

16. Villa AD, Sammut E, Zarinabad N, Carr-White G, Lee J, Bettencourt N, et al. Microvascular ischemia in hypertrophic cardiomyopathy: new insights from high-resolution combined quantification of perfusion and late gadolinium enhancement. J Cardiovasc Magn Reson. 2016;18:4

17. Sciagra R, Calabretta R, Cipollini F, Passeri A, Castello A, Cecchi F, et al. Myocardial blood flow and left ventricular functional reserve in hypertrophic cardiomyopathy: a (13)NH3 gated PET study. Eur J Nucl Med Mol Imaging. 2017;44(5):866-75

18. Kao YC, Lee MF, Mao CT, Chen WS, Yang NI, Cherng WJ, et al. Differences of left ventricular systolic deformation in hypertensive patients with and without apical hypertrophic cardiomyopathy. Cardiovasc Ultrasound. 2013;11:40.

19. Urbano-Moral JA, Rowin EJ, Maron MS, Crean A, Pandian NG. Investigation of global and regional myocardial mechanics with 3-dimensional speckle tracking echocardiography and relations to hypertrophy and fibrosis in hypertrophic cardiomyopathy. Circ Cardiovasc Imaging. 2014;7(1):11-9.

Ready to submit your research? Choose BMC and benefit from:

- fast, convenient online submission

- thorough peer review by experienced researchers in your field

- rapid publication on acceptance

- support for research data, including large and complex data types

- gold Open Access which fosters wider collaboration and increased citations

- maximum visibility for your research: over $100 \mathrm{M}$ website views per year

At $\mathrm{BMC}$, research is always in progress.

Learn more biomedcentral.com/submissions 